\title{
8. From knowledge transfer to knowledge sharing? Towards better links between research, policy and practice
}

\author{
Brian Head
}

\section{Introduction}

Why is there such a wave of interest in the processes of knowledge transfer, knowledge translation and knowledge brokering? What social and organisational problems seem to require these processes? We all believe that better knowledge, wider understanding and enhanced cooperation will generally lead to better outcomes. Achieving these broad objectives is, however, difficult. In the modern world, we suffer from the dilemma of being information rich and time poor. We are often aware that others have important insights to complement our own perspectives, but the transaction costs of access and engagement are often so high that more cooperative approaches to knowledge and action are doomed to failure. How, then, can we find economical and effective ways to broaden our knowledge and improve our capacity for joint action to tackle major issues?

One common approach is to promote the 'transfer' or 'transmission' of knowledge from one group or sector to others. This is what I call a knowledgetransmission approach. The language of 'knowledge transmission' can, however, sometimes imply that some people (experts) have access to important truths that need to be learned, accepted, adopted and implemented by others. Indeed, there are numerous situations where a didactic approach is highly appropriate and efficient. A single authoritative framework is useful and even essential for training and guidance activities where precision and consistency are the top priorities. Examples include the standard operating procedures required to undertake a financial audit; to operate technical equipment; to ensure quality control on a pharmaceutical production line; or to administer clinical procedures such as immunisation. The knowledge generated by scientific expertise is not, however, always simple, readily codified and transferable in this way. Hence the knowledge-transmission model needs to be expanded. 
First, expertise tends to grow around disciplinary cultures and professional organisations with their own specialised languages and conceptual frameworks. It is difficult for most people to understand a wide span of disciplinary and professional knowledge, which sometimes has competing as well as complementary approaches to similar issues. Knowledge translation is the activity of working to increase understanding across disciplines or professional boundaries. Here, the insights of one group are elucidated and packaged for other audiences.

Second, scientific-technical expertise cannot guarantee general consensus about how problems should be defined and resolved. Thus, there is disagreement on the nature of the problems, the development of preferred solutions and how practical interventions can be effectively implemented. Knowledge brokering focuses on harnessing the diverse insights of the professions and academic disciplines around key problems of understanding and action. This approach is by no means straightforward, for reasons outlined below.

\section{Fragmentation and complexity}

In the real world, the understanding of issues and problems can often be hotly contested, with a divergence of viewpoints and recommended pathways. This usually arises for two main reasons. The first is the fragmentation of perspectives and social understandings, associated with competing value preferences, different occupations, social roles, organisational contexts and knowledge disciplines. The second reason is the inherently complex and multi-layered nature of many important problems that attract our attention. Many of these problems arise from multiple causes and are interconnected. For example, there is no simple cause and thus no simple remedy for low educational achievement, child poverty, preventable diseases and addictions, Indigenous disadvantage, ecological degradation, and so on. Hence, as a result of these two factorsfragmentation and complexity-an inclusive and connected approach to knowledge and action is required. It is useful to begin by recognising the different foundations of relevant knowledge.

The scientific-technical approach to producing systematic knowledge (with its rigorous protocols for data collection, data quality and testing of causal explanations) provides enormously valuable explanations and insights. Indeed, there could be several relevant disciplinary streams contributing to the scientific knowledge base of a problem. For example, research into drugs and alcohol has benefited from the contributions of criminology, economics, sociology, public health, psychology and neurobiological sciences. Scientific-technical knowledge is, however, only one contributor to the 'knowledge' segment of 
the knowledge--policy-practice relationship. Sole reliance on rigorous scientific knowledge is impractical, especially since some approaches (for example, the experimental sciences and data modelling) are somewhat disconnected from the 'real world' of professional practice and policy development.

Thus, in addition to scientific-technical knowledge, other important knowledge contributions arise from the professionals and practitioners who manage programs, deliver services and assist clients. This realm of 'practice' knowledge is rich, diverse and enlivened by the practitioners' familiarity with numerous cases, clients and contexts. Practitioner knowledge, at its best, is alive to situational experience (McAdam et al. 2007; Schon 1983; Wenger 1998), but develops in professional silos. The world of 'practice wisdom', shared and communicated among practitioners, is not typically characterised by field trials and rigorous testing of data quality. Nevertheless, there are many pressures, especially in contemporary health and social care programs, for practitioners to follow 'best-practice' codes and procedures, shaped by the need for professional accountability and informed by the findings of scientific research on the efficacy of various approaches (Mullen et al. 2008).

The third realm of relevant knowledge is policy development and its link to political accountability - the realm of politicians and senior public servants. Policymakers do not simply follow and adopt the findings of scientific research and professional practice. Rather, policymakers make use of a wide range of information and ideas, as part of their broad consideration of arguments for and against policy adjustment, options for the balancing of interests and calculations about support and legitimacy (Bowen and Zwi 2005; Head 2008a).

A fourth realm of relevant knowledge is the direct experience of citizens who are users of services - for example, stakeholders involved as clients, carers or advocates in local community organisations. Their views can easily be neglected or overlooked, unless given formal weight through the consultation and evaluation processes of a program review or publicised through advocacy activities in the media.

\section{The rise of collaborative networks}

Research, policy and practice tend to operate with different concerns, languages and time lines (Brownson et al. 2006; Lomas 1990; Shonkoff 2000). In this sense, knowledge is 'sticky' and contextual and few individuals have the skills and time to 'translate' knowledge across these disciplinary and organisational divides.

In a number of areas of human development and human services, professionals and informed stakeholders have concluded that complex issues of wellbeing 
are not amenable to technical solutions by a set of experts. These big issues can best be tackled by collective efforts in which representatives of research, policy and practice pool their scientific and practical knowledge about what needs to be done. Accordingly, collaborative networks (such as those funded in recent years by the Australian Research Council) have developed forums to facilitate dialogue and consensus between researchers, policymakers and practitioners about research priorities and the implications of research findings for more effective interventions (Head and Stanley 2007). The emphasis is less on basic research (that is, identifying and filling 'gaps' in knowledge) and more on mobilising the best available applied knowledge, drawn from various sectors.

The network approach has identified the need for much improved interaction and communication across the policy, practice and research sectors. For example, how can the fruits of applied research be disseminated in more accessible forms that can be understood and utilised by policymakers and practitioners (Feldman and Kane 2003; Lewig et al. 2006)? Equally, how can the tacit knowledge of practitioners be made explicit and shared more widely (McAdam et al. 2007)? In other words, how can the insights of 'practice wisdom' be collated and communicated to inform the work of researchers and policymakers (Salveron et al. 2006; Schorr 2003)? In fact, the communication of knowledge needs to be multi-directional, so that all sectors are better informed about the specific needs and approaches of the others. This allows for mutual understanding and mutual adjustment. Collaborative networks have the objective of knowledge sharing across sectors and disciplines as a basis for more effective policy frameworks and service systems. The question then becomes: what are the most effective approaches to better interaction and mutual understanding?

Although there could be a shared desire to address program/service improvement, the problem of professional silos and fragmentation of knowledge leads to a dispersion of uncoordinated effort. It follows that strategies are needed for promoting interaction between individuals from all relevant sectors. The initial purposes of such interaction are to improve flows of information, improve mutual understanding and thus improve relationships and trust. Production and circulation of documentation are important for mutual understanding, further analysis and for advocacy purposes. Documents alone, however, are insufficient; face-to-face contact seems to be the best way to ensure that relevant insights and knowledge are provided to those who need them in each sector (Lomas 2000). 


\section{From interaction to strategic change}

Given the entrenched difficulties associated with the establishment of knowledge sharing and brokering across sectors and disciplines, many different activities and techniques are being pursued to promote useful interaction and sharing of ideas (Tsui 2006). These might be understood as ranging in intensity from casual interaction through to regular structured dialogue and engagement around major projects. As informal networks of interaction are developed, a potential basis is built for cooperative activities into the future. Networks can give rise to crosssectoral teams focusing on specific needs and challenges, perhaps in a local area. Interest networks on particular topics can be established with participation from all sectors. These could operate at a local, regional or even national scale depending on access to communications technology. Networks of interaction are useful and necessary, but more fundamental types of behavioural change are also necessary within each sector to bridge the disciplines and accelerate strategic actions across the sectors to tackle major social problems.

Some challenges and issues for policymakers, researchers and practitioners are briefly noted below.

For policymakers, the key questions might include

- are we working on the most important problems (have we taken account of the views of clients and professional service providers, as well as our political leaders)

- are we seeking to expand the 'space' available inside public agencies to learn, reflect and develop new approaches

- are we seeking to involve practitioners more deeply in the design, delivery and evaluation of programs to maximise their effectiveness

- are we taking full account of cultural contexts in designing programs for Indigenous people (cf. Larkin 2006)

- are we building evaluation into the foundations of all programs, and are we taking the opportunities to learn from these findings even when the results are discomforting?

For the research sector, some of the key questions might include

- are we working on the most important problems (have we taken account of the views of policymakers, practitioners and program clients)

- have we systematically involved other sectors (who will be users of and audiences for research) in our consideration of the knowledge base for assessing program effectiveness 
- are we working at an appropriate scale (micro or macro; local or regional; single or multiple issues) that will be of interest to policy and practice partners

- have we summarised our research findings in ways that draw out the implications for practitioners and decision makers

- will our findings be accessible and intelligible to other sectors and to the media?

For the practitioner sector, some of the key questions might include

- are we taking steps to document the impacts of our work with particular groups of clients as a basis for collective discussion of evidence-based practice (cf. Mullen et al. 2008; Simons 2004)

- are we creating or using frameworks for linking client casework to broader program and policy considerations (cf. Proctor and Rosen 2008)

- are we gathering evidence concerning the interlinked nature of social problems and successful measures for addressing such complex issues

- are we utilising and strengthening community networks on key issues (cf. Foster-Fishman et al. 2001)

- are we utilising new forums for continuing review of 'best-practice' approaches and attention to 'prospective' new approaches

- are we inviting policy and research colleagues into these forums?

\section{Tackling big issues through collaboration}

It has been demonstrated in many contexts that professional knowledge bases are the important building blocks of improved understanding, but that the 'silos' of professional knowledge are also obstacles to the diffusion of innovation (Dopson and Fitzgerald 2005; Ferlie et al. 2005). I have argued that if the challenge for collective engagement is to focus on high-priority complex issues then the task goes well beyond casual networking and information exchange. This is a strategic challenge requiring large-scale resources and collective energy, a focus on agenda setting and moving from knowledge to action. Pooling the insights of diverse experts from each sector (research, policy, practice) is only the beginning of a collaborative process that requires a significant degree of coordination (see Figure 8.1). For example, the Australian Research Alliance for Children and Youth (ARACY 2004) 'collaboration framework' envisages a carefully designed process among key players. This collaborative process would 
require the participants to define key issues, take stock of expert knowledge, evaluate the impediments to progress, recommend fresh approaches and unpack the implications for policy, practice and research.

\section{Figure 8.1 The interactive hub of collaboration}

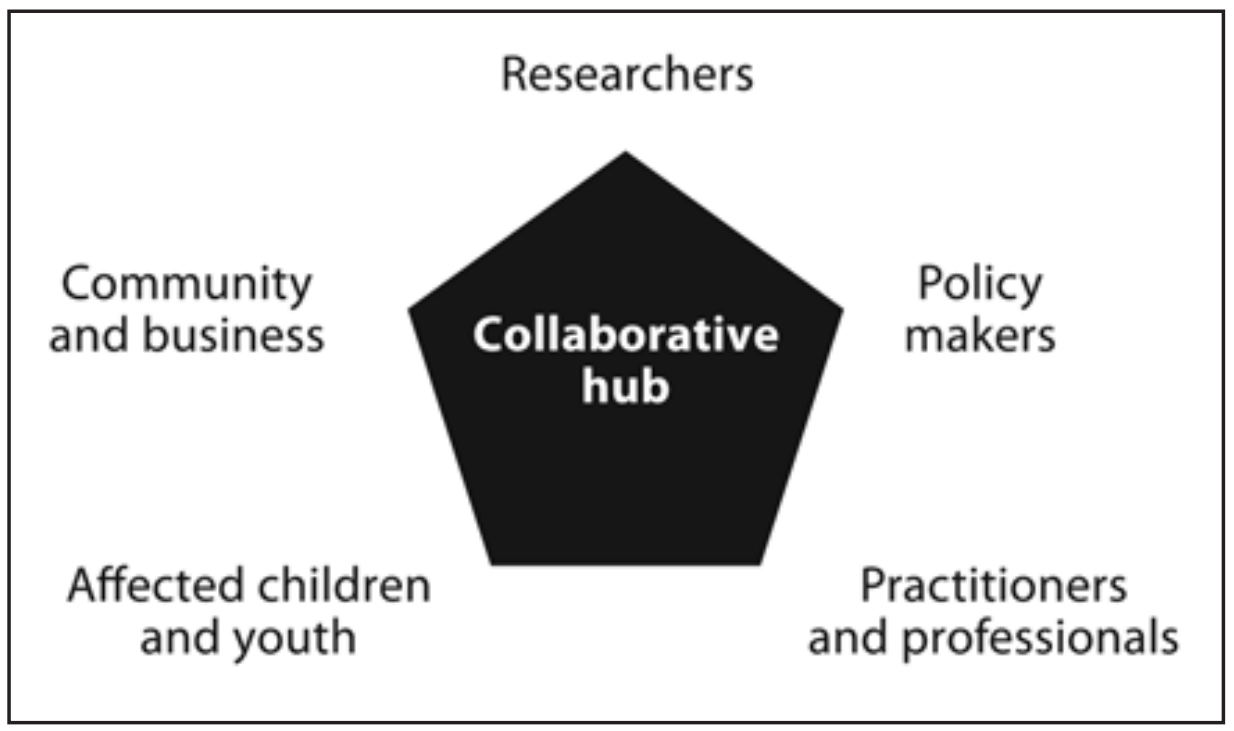

Patterns of knowledge sharing across the sectors (research-policy-practice) need to be appropriate to the specific contexts of joint work. It is necessary to construct these relationships in ways appropriate for each problem context and to recognise that partnering arrangements can be constructed in diverse ways. In principle, the focus of joint work could be highly varied, such as

- devising and testing different 'scenarios' for the use of knowledge and strategy in pursuit of policy objectives (cf. Oreszczyn and Carr 2008)

- field testing a pilot program, delivered by a mix of non-governmental organisations (NGOs) and public agencies, funded by government and evaluated by researchers

- documenting the outcomes of a mature program devised and delivered by NGOs, funded by corporate philanthropy and initially evaluated by consultants

- fundamental reassessment of why government programs for Indigenous communities have so often failed (requiring a sustained 'think tank' forum informed by many forms of knowledge)

- documenting and disseminating promising examples of local initiatives, perhaps led by practitioners, that appear to have produced positive outcomes 
- assessing the strengths and limitations of inter-agency coordination and intergovernmental cooperation as strategic frameworks for the improvement of government services.

The knowledge-sharing challenges in each of these examples are rather different. Knowledge-sharing strategies and processes can be judged as more or less 'effective' only in relation to stated purposes. What works well for one purpose, or in one context, might be insufficient elsewhere. One example of effective knowledge sharing is the Inala Program in south-east Queensland, where an innovative prevention and support program was designed and implemented through a partnership between government agencies, a community-based NGO (Mission Australia) and a university research team (Freiberg et al. 2005; Homel et al. 2006). The focus on developmental prevention and early intervention for a group of preschool children from disadvantaged families required close collaboration between the children and their parents/carers, the teachers, professionals providing support services, government agency staff and the research team. This multi-layered approach has produced some positive outcomes, which in turn influenced the design of other national and regional programs focusing on the early years and readiness for school.

The common intent in all such initiatives is to establish shared ownership of new thinking and new strategies. Knowledge brokering can play a vital role. It involves active strategies among relevant actors for sharing and negotiating understandings about problems, evidence bases and solutions. Building strong relationships and participatory processes is essential for knowledge brokering.

Much has been written about the growing role of influential individuals who provide a service in bridging and linking between groups or institutions. These individuals have a capacity to build bridges, span boundaries and otherwise facilitate the translation and adoption of ideas (for example, Tsui 2006; Williams 2002). In an increasingly networked and mediated environment, linking and bridging roles are common and necessary. Innovative organisations will increasingly seek to embed such roles in their senior teams to promote productive interchange and partnering. While there are real benefits from interactive and collaborative approaches, there are, however, also costs.

Investing in knowledge sharing across disciplines and sectors has real costs, especially in higher transaction costs of collaborative approaches. These are centred primarily on the time and effort required for engagement, communication and goal-setting discussions across disciplines and sectors, which are a necessary prerequisite to project planning and implementation (Metcalfe et al. 2006). Similarly, an organisation contemplating various cooperative options 
for pursuing its goals will need to assess whether the potential benefits are outweighed by the administrative and communication costs of 'cooperation' with others (White 2005).

Partly in response to this issue, a new breed of network organisations has emerged (such as ARACY), dedicated to knowledge brokering and knowledge sharing on behalf of their members. This network format provides an economy of collective effort to reap some of the rewards of collective action. The ideal situation might be that every organisation in each sector would redesign its internal arrangements in order to improve its communications, participate in regular dialogue with others and share in new processes of joint thinking and action. It is, however, unrealistic to expect all organisations to develop new capacities for innovative and shared thinking. Involvement in network organisations is therefore a useful and convenient way to take some first steps towards these new capacities. Assisting in the establishment of new forums for the latter activities is an indicator of how far such organisations might have changed.

Many groups are also undertaking more specific forms of partnering activities. Longer-term partnerships are needed to build trust around high-quality research on matters of mutually agreed importance. A successful partnership of this type will focus on the 'co-production' of useful knowledge. Interchange of personnel might be useful to promote the exchange of ideas and understandings; and cross-appointments to the advisory bodies of other organisations can also assist. Research partnerships - for example, Australian Research Council (ARC) Linkage grants - can be useful. Contract research involving several parties also has inherent risks - for example, delays and complexities arising from the need for multi-party contractual agreements; the lack of continuity of key staff (rapid turnover in policy and program positions can undermine a coherent sense of direction); and the long time frames before the delivery of usable results.

\section{Dilemmas for research relevance}

There is increasing pressure for academic researchers to ensure their applied work is more responsive and attuned to the perspectives of other sectors (Davies et al. 2000; Nutley et al. 2007). Social research is of a higher standard when it takes account of the views of practitioners and policymakers. For example, if social research projects ignore the views of practitioners and service providers, such analyses are likely to be deficient, since they are unlikely to be alert to the success factors underlying effective interventions. And yet, the incentive structures and rewards for academic research give priority to prestigious publications and success in competitive grants. These criteria of success tend to 
work against giving higher priority to social relevance. (A partial exception is in the medical or technical sciences to the extent that new insights can be linked to commercial applications.)

In the human services and the social care sectors, there has been a huge research literature establishing the key risk factors and protective factors for the healthy development of young people. The shift from knowledge to action is, however, constrained at many points. Programs based on technical advances (for example, immunisation) are readily implemented. More complex programs, however, addressing multiple related problems have been difficult to design and implement with long-term funding. The importance of proceeding cautiously through local pilot projects and local evaluations is well established. Nevertheless, it has been difficult to develop large-scale programs that are well informed by continuing social research and feedback from practical experience. This is perhaps the greatest challenge for research relevance and perhaps the greatest potential contribution of a collaborative model for cross-sector strategy development.

If policymakers believe that academic research is abstract or irrelevant, academic researchers will be further sidelined and the research consultancy industry will win most of the applied social research contracts. If, however, social research 'over-compensates' by becoming highly instrumental - tailoring research to fit the current requirements of a funding agency - this also has major dangers. Academic research is increasingly required to demonstrate two forms of value that pull in different directions: excellence in quality/rigour and relevance to the priority concerns of funders and stakeholders. The ideal outcome would be high-quality research on topics of high importance. Importantly, however, judgments concerning the impact or relevance of research will continue to depend on the views of other sectors - government, business, community, and so on. If a major goal of researchers is to increase the utilisation of research findings (Amara et al. 2004; Huberman 1994; Weiss 1979), this would require researchers to take an active approach that goes beyond mere dissemination or diffusion of research papers (Lavis et al. 2003). Active marketing, engagement and influencing would be necessary, but not just around a research report. The most powerful methods for long-term influence are likely to be knowledgesharing approaches built around major continuing themes or problems.

\section{Conclusions}

Shared thinking comes from a partnering approach. We have noted that personal relationships and face-to-face interactions are very important and should be encouraged. Even more significant, however, is the need to boost the sense of 
'ownership' of applied research directions and findings. Research itself requires a more integrated approach (Bammer 2006). Moving towards a co-production model, new thinking for joint research could extend to designing research questions about policy and practice jointly among the stakeholders, funders and representatives of service users.

The development of more strategic approaches to service improvement requires new network arrangements to promote better communication and shared perspectives across the professions and across the policy-research-practice sectors (Scott and Thurston 2003; Wandersman et al. 2008; Weinstein et al. 2003). For these new forums to have greater impact, it is important to encourage the growth of 'learning cultures' within organisations as well as across organisations. Hence it is necessary to influence the organisational climate within agencies at a senior level, so that they are more receptive to dialogue about new approaches and more receptive to the findings of jointly agreed research programs. Similar changes are necessary in the research cultures of academic and non-governmental organisations.

The process requirements for successful collaboration seem to include inclusive participation, mutual respect for each other's knowledge and values, support for members to participate fully in the broader network, clarity on the rules for joint decision making and effective leadership roles. The sustainability of networkstheir capacity to thrive and adapt over a long period — is important (Head 2006). Collaborative networks seem to be the best method currently available to address the inherent complexity and fragmentation embedded in social problems and the best way to achieve outcomes in a contested policy environment (Head 2008 b). The criteria for judging the effectiveness of collaborative networks are, however, likely to be divergent, owing to the different views and interests of participants (Provan and Milward 2001). In a networked world, knowledge transmission is no longer sufficient to generate a new consensus around goals and strategies. We need to develop our knowledge-brokering and knowledgesharing capabilities to tackle the big strategic issues we face.

\section{References}

Amara, N., Ouimet, M. and Landry, R. 2004, 'New evidence on instrumental, conceptual, and symbolic utilization of university research in government agencies', Science Communication, vol. 26, no. 1, pp. 75-106.

Australian Research Alliance for Children and Youth (ARACY) 2004, ARACY Framework for Collaboration, Australian Research Alliance for Children 
Bridging the 'Know-Do' Gap

and Youth, Canberra, viewed 3 May 2010, <http://www.aracy.org.au/ publicationDocuments/TOP_ARACY_Framework_for_Collaboration_2005. pdf $>$

Australian Research Alliance for Children and Youth (ARACY) 2008, Report Card on the Wellbeing of Young Australians, Australian Research Alliance for Children and Youth, Canberra.

Bammer, G. 2006, 'A systematic approach to integration in research', Integration Insights, no. 1, ANU College of Medicine and Health Sciences, Canberra, viewed 3 May 2010, <http://i2s.anu.edu.au/sites/default/files/integrationinsights/integration-insight_l.pdf>

Bowen, S. and Zwi, A. B. 2005, 'Pathways to "evidence-informed" policy and practice: a framework for action', PLoS Medicine, vol. 2, no. 7 (el66), pp. $100-5$.

Brownson, R. C., Royer, C., Ewing, R. and McBride, T. D. 2006, 'Researchers and policymakers: travelers in parallel universes', American Journal of Preventive Medicine, vol. 30, no. 2, pp. 164-72.

Davies, H., Nutley, S. and Smith, P. (eds) 2000, What Works? Evidence-based policy and practice in public services, Policy Press, Bristol.

Dopson, S. and Fitzgerald, L. (eds) 2005, Knowledge to Action? Evidence-based health care in context, Oxford University Press, UK.

Feldman, P. H. and Kane, R. L. 2003, 'Strengthening research to improve the practice and management of long-term care', Milbank Quarterly, vol. 81, no. 2, pp. 179-220.

Ferlie, E., Fitzgerald, L., Wood, M. and Hawkins, C. 2005, 'The nonspread of innovations: the mediating role of professionals', Academy of Management Journal, vol. 48, no. 1, pp. 117-34.

Foster-Fishman, P., Berkowitz, S., Lounsbury, D., Jacobson, S. and Allen, N. 2001, 'Building collaborative capacity in community coalitions: a review and integrative framework', American Journal of Community Psychology, vol. 29, no. 2 , pp. $241-61$.

Freiberg, K., Homel, R., Batchelor, S., Carr, A., Hay, I. Elias, G., Teague, R. and Lamb, C. 2005, 'Pathways to participation: a community-based developmental prevention project in Australia', Children and Society, vol. 19, no. 2, pp. 14457. 
Head, B. W. 2006, Effective Collaboration, July, Australian Research Alliance for Children and Youth, Canberra, viewed 3 May 2010, <http://www.aracy.org. au/publicationDocuments/TOP_Effective_Collaboration_2006.pdf>

Head, B. W. 2008a, 'Three lenses of evidence based policy', Australian Journal of Public Administration, vol. 67, no. 1, pp. 1-11.

Head, B. W. 2008b, 'Assessing network-based collaborations: effectiveness for whom?', Public Management Review, vol. 10, no. 6, pp. 733-49.

Head, B. W. and Stanley, F. J. 2007, 'Evidence-based advocacy', International Journal of Adolescent Medicine and Health, vol. 19, no. 3, pp. 255-62.

Homel, R., Freiberg, K., Lamb, C., Leech, M., Carr, A., Hampshire, A., Hay, I., Elias, G., Manning, M., Teague, R. and Batchelor, S. 2006, The Pathways to Prevention Project: The first five years, 1999-2004, Mission Australia and Key Centre for Ethics, Law, Justice and Governance, Griffith University, Brisbane.

Huberman, M. 1994, 'Research utilization: the state of the art', Knowledge and Policy: The international journal of knowledge transfer and utilization, vol. 7, no. 4, pp. 13-33.

Larkin, S. 2006, 'Evidence-based policy making in Aboriginal and Torres Strait Islander health', Australian Aboriginal Studies, vol. 2, pp. 17-26.

Lavis, J. N., Robertson, D., Woodside, J. M., McLeod, C. B. and Abelson, J. 2003, 'How can research organizations more effectively transfer research knowledge to decision makers?', Milbank Quarterly, vol. 81, no. 2, pp. 221-48.

Lewig, K., Arney, F. and Scott, D. 2006, 'Closing the research-policy and research-practice gaps: ideas for child and family services', Family Matters, vol. 74, pp. 12-19.

Lomas, J. 1990, 'Finding audiences, changing beliefs: the structure of research use in Canadian health policy', Journal of Health Politics, Policy and Law, vol. 15, no. 3, pp. 525-42.

Lomas, J. 2000, 'Using "linkage and exchange" to move research into policy at a Canadian foundation', Health Affairs, vol. 19, no. 3, pp. 236-40.

McAdam, R., Mason, B. and McCrory, J. 2007, 'Exploring the dichotomies within the tacit knowledge literature: towards a process of tacit knowing in organizations', Journal of Knowledge Management, vol. 11, no. 2, pp. 43-59.

Metcalfe, J., Riedlinger, M., Pisarski, A. and Gardner, J. 2006, Collaborating Across the Sectors, Council for the Humanities, Arts and Social Sciences, Canberra. 
Bridging the 'Know-Do' Gap

Mullen, E. J., Bledsoe, S. E. and Bellamy, J. L. 2008, 'Implementing evidencebased social work practice', Research on Social Work Practice, vol. 18, no. 4, pp. 325-38.

Nutley, S., Walter, I. and Davies, H. 2007, Using Evidence: How research can inform public services, Policy Press, Bristol.

Oreszczyn, S. and Carr, S. 2008, 'Improving the link between policy research and practice', Qualitative Research, vol. 8, no. 4, pp. 473-97.

Proctor, E. and Rosen, A. 2008, 'From knowledge production to implementation', Research on Social Work Practice, vol. 18, no. 4, pp. 285-91.

Provan, K. G. and Milward, H. B. 2001, 'Do networks really work? A framework for evaluating public sector organisational networks', Public AdministrationReview, vol. 61, no. 4, pp. 414-23.

Salveron, M., Arney, F. and Scott, D. 2006, 'Sowing the seeds of innovation: ideas for child and family services', Family Matters, vol. 73, pp. 39-45.

Schon, D. A. 1983, The Reflective Practitioner: How professionals think in action, Basic Books, New York.

Schon, D. A. and Rein, M. 1994, Frame Reflection: Toward the resolution of intractable policy controversies, Basic Books, New York.

Schorr, L. B. 2003, Determining 'What Works' in Social Programs and Social Policies: Toward a more inclusive knowledge base, Brookings Institution, Washington, DC.

Scott, C. M. and Thurston, W. E. (eds) 2003, Collaboration in Context, University of Calgary, Alberta.

Shonkoff, J. P. 2000, 'Science, policy, and practice: three cultures in search of a shared mission', Child Development, vol. 71, no. 1, pp. 181-7.

Simons, H. 2004, 'Utilizing evaluation evidence to enhance professional practice', Evaluation, vol. 10, no. 4, pp. 410-29.

Tsui, L. 2006, A Handbook for Knowledge Sharing, Community-University Partnership for Study of Children Youth and Families, Alberta.

Wandersman, A., Duffy, J., Flaspohler, P., Noonan, R., Lubell, K., Stillman, L., Blachman, M., Dunville, R. and Saul, J. 2008, 'Bridging the gap between prevention research and practice', American Journal of Community Psychology, vol. 41, nos 3-4, pp. 171-81. 
Weinstein, J., Whittington, C. and Leiba, T. (eds) 2003, Collaboration in Social Work Practice, Jessica Kingsley, London.

Weiss, C. H. 1979, 'The many meanings of research utilization', Public Administration Review, vol. 39, no. 5, pp. 426-31.

Wenger, E. 1998, Communities of Practice, Cambridge University Press, UK.

White, S. 2005, 'Cooperation costs, governance choice and alliance evolution', Journal of Management Studies, vol. 42, no. 7, pp. 1383-412.

Williams, P. 2002, 'The competent boundary spanner', Public Administration, vol. 80, no. 1, pp. 103-24. 